\title{
A Histone Deacetylase, MoHDA1 Regulates Asexual Development and Virulence in the Rice Blast Fungus
}

\author{
Taehyun Kim ${ }^{1 \dagger}$, Song Hee Lee ${ }^{1,2 \dagger}$, Young Taek $\mathrm{Oh}^{3}$, and Junhyun Jeon (iD) $1,2 *$ \\ ${ }^{1}$ Department of Biotechnology, College of Life and Applied Sciences, Yeungnam University, Gyeongsan 38541, Korea \\ ${ }^{2}$ Plant Immunity Research Center, Seoul National University, Seoul 08826, Korea \\ ${ }^{3}$ Freshwater Bioresources Utilization Division, Nakdonggang National Institute of Biological Resources, Sangju 37242, \\ Korea
}

(Received on June 9, 2020; Revised on July 14, 2020; Accepted on July 19, 2020)

Interplay between histone acetylation and deacetylation is one of the key components in epigenetic regulation of transcription. Here we report the requirement of MoHDA1-mediated histone deacetylation during asexual development and pathogenesis for the rice blast fungus, Magnaporthe oryzae. Structural similarity and phylogenetic analysis suggested that MoHDA1 is an ortholog of Saccharomyces cerevisiae Hda1, which is a representative member of class II histone deacetylases. Targeted deletion of MoHDA1 caused a little decrease in radial growth and large reduction in asexual sporulation. Comparison of acetylation levels for H3K9 and H3K14 showed that lack of MoHDA1 gene led to significant increase in $\mathrm{H} 3 \mathrm{~K} 9$ and $\mathrm{H} 3 \mathrm{~K} 14$ acetylation level, compared to the wild-type and complementation strain, confirming that it is a bona fide histone deacetylase. Expression analysis on some of the key genes involved in asexual reproduction under sporulation-promoting condition showed almost no differences among strains, except for MoCON6 gene, which was up-regulated more than 6-fold in the mutant than wild-type. Although the deletion mutant displayed little defects in germination and

\footnotetext{
${ }^{\dagger}$ These authors contributed equally to the work.

*Corresponding author.

Phone) +82-53-810-3030, FAX) +82-53-810-4769

E-mail) jjeon@yu.ac.kr

ORCID

Junhyun Jeon

https://orcid.org/0000-0002-0617-4007

(c) This is an Open Access article distributed under the terms of the Creative Commons Attribution Non-Commercial License (http:// creativecommons.org/licenses/by-nc/4.0) which permits unrestricted noncommercial use, distribution, and reproduction in any medium, provided the original work is properly cited.
}

Articles can be freely viewed online at www.ppjonline.org. subsequent appressorium formation, the mutant was compromised in its ability to cause disease. Woundinoculation showed that the mutant is impaired in invasive growth as well. We found that the mutant was defective in appressorium-mediated penetration of host, but did not lose the ability to grow on the media containing $\mathrm{H}_{2} \mathrm{O}_{2}$. Taken together, our data suggest that MoHDA1-dependent histone deacetylation is important for efficient asexual development and infection of host plants in M. oryzae.

Keywords : class II HDAC, MoHDA1, rice blast fungus

Handling Editor : Sook-Young Park

Deacetylation of lysine residues in histones is one of the major epigenetic mechanisms through which eukaryotic cells regulate transcription (Bannister and Kouzarides, 2011; Jeon et al., 2014). Generally, histone deacetylation in conjunction with concomitant histone methylation is associated with repression of genes, whereas high levels of histone acetylation correlates with active transcription of genes, although there are a few exception to this rule (Bannister and Kouzarides, 2011). Several decades of works have shown that histone acetylation is a reversible epigenetic mark regulated by antagonistic interplay between two groups of enzymes - histone acetyltransferases and histone deacetylases (HDACs) (Lee and Workman, 2007; Seto and Yoshida, 2014).

HDACs belong to either the HDAC family or the Sir2 family, and are traditionally divided into separate categories called classes (class I to class IV) according to sequence similarities and type of co-factors required for enzyme activity (Seto and Yoshida, 2014). HDAC fam- 
ily consist of class I, II, and IV HDACs, which require zinc ions as co-factors, while Sir2 family contains class III HDACs, which require $\mathrm{NAD}^{+}$as a co-factor for removal of acetyl group from lysine residues. Among different classes of HDACs, Hdal is a founding member of class II HDACs that contains the corresponding deacetylase domain and the Arb2 domain, latter of which is required for H3K9 methylation (Seto and Yoshida, 2014; Shen et al., 2016). Several studies have examined the roles of Hda1-mediated histone deacetylation in yeast such as Saccharomyces cerevisiae and Candida albicans (Carmen et al., 1996; Robbins et al., 2012; Rundlett et al., 1996; Yang and Seto, 2008). In $S$. cerevisiae and Schizosaccharomyces pombe, it was shown that Hda1 is required for progression of cell cycle and developmental events as well as transcriptional regulation (Carmen et al., 1996; Rundlett et al., 1996). In C. albicans, Hda1 has been demonstrated to play a key role in the regulation of filamentous growth, switching between the white and opaque states, and virulence (Srikantha et al., 2001; Zacchi et al., 2010).

Specific roles of some HDACs such as Rpd3 and Hos2, all of which are Class I enzymes, during pathogenesis have been reported in different plant pathogenic fungi to date (Elias-Villalobos et al., 2019; Jeon et al., 2014). In Fusarium graminearum, for example, $H D F 1$, an ortholog of Hos2, was demonstrated to be involved in spore formation, production of mycotoxins such as deoxynivalenol, and plant infection (Li et al., 2011). In Ustilago maydis, Hos 2 was shown to regulate virulence via its regulation of mating-type genes (Elias-Villalobos et al., 2015). In the rice blast fungus, Magnaporthe oryzae (Pyricularia oryzae), MoHOS2 (M. oryzae HOS2), which was identified as a interacting protein with TIG1 as a components in Set3 complex (Ding et al., 2010), was shown to be required for asexual development and pathogenicity (Lee et al., 2019). In contrast to extensive studies on class I HDACs, the class II HDACs have received little attention, although the Clr3, a homolog to $S$. cerevisiae Hda1 and $S$. pombe $\mathrm{Clr} 3$, in $U$. maydis was reported to contribute to virulence (EliasVillalobos et al., 2019). Here we investigated the roles of MoHDA1 as a HDAC in fungal development and pathogenesis in the rice blast fungus, $M$. oryzae.

M. oryzae is a causal agent of the rice blast disease, which has socio-economic importance in rice-cultivating regions (Dean et al., 2012; Talbot, 2003). Infection process of the rice blast fungus begins with dispersal of asexual spores called conidia that land and germinate on rice leaf surface. Upon sensing the environmental cues such as surface hydrophobicity, germ tube tip differentiates into appressorium, a specialized infection cell. A multitude of signaling pathways involving cAMP, calcium and MAP kinases has been shown to regulate such developmental processes (Howard and Valent, 1996; Jiang et al., 2018; Ryder and Talbot, 2015; Wilson and Talbot, 2009). Turgor pressure up to $8 \mathrm{MPa}$ within the appressorium allows the fungus to drive penetration hyphae, through the cuticular layer of plant, into the underlying epidermal cells (Wilson and Talbot, 2009). Colonization of host tissues with ramifying, bulbous invasive hyphae ensues immediately from penetration, consequently leading to destruction of young seedling or grain-bearing structures of adult plants (Fernandez and Orth, 2018). During such intimate interaction between host and pathogen, host plants deploy defense responses including production of reactive oxygen species (ROS), whereas the fungal pathogen secretes diverse array of apoplastic and cytoplasmic effector proteins to subvert such defense responses and/or to manipulate host metabolism for nutrient acquisition and invasive growth (Zhang and $\mathrm{Xu}, 2014$ ).

In this study, we provide evidence that MoHDA1 is a bona fide HDAC targeting $\mathrm{H} 3 \mathrm{~K} 9 \mathrm{ac}$ and $\mathrm{H} 3 \mathrm{~K} 14 \mathrm{ac}$, and that such deacetylation activity is particularly important for asexual spore production and virulence.

\section{Materials and Methods}

Fungal isolates and culture conditions. The field isolate KJ201, which was provided by the Fungal Genetic Resource Center (CFGR, http://genebank.snu.ac.kr), was used as a wild-type strain throughout the work. Mycelia used for DNA, RNA extraction, and protoplast preparation was cultured on liquid complete medium $(0.6 \%$ yeast extract, $0.6 \%$ tryptone and $1 \%$ sucrose; all w/v) at $25^{\circ} \mathrm{C}$ and 120 rpm. All strains used for growth comparison and preparation of spore suspension were grown in complete agar medium $(0.6 \%$ yeast extract, $0.6 \%$ tryptone, $1 \%$ sucrose and $1.5 \%$ agar powder; all $\mathrm{w} / \mathrm{v}$ ) and oatmeal agar medium for 9 days at $25^{\circ} \mathrm{C}$. Fungal cultures used to test for sensitivity to $\mathrm{H}_{2} \mathrm{O}_{2}$ and lysing enzymes were grown at $25^{\circ} \mathrm{C}$ for 9 days in complete media plates containing a final concentration of $1 \mathrm{mM}, 3 \mathrm{mM}$, and $5 \mathrm{mM} \mathrm{H}_{2} \mathrm{O}_{2}$, a final concentration of $5 \mathrm{mg} / \mathrm{ml}$ of lysing enzyme and both. Selection of hygromycin-resistant transformants was carried out using TB3 agar plates $[0.3 \%$ yeast extract $(\mathrm{w} / \mathrm{v}), 0.3 \%$ casamino acid $(\mathrm{w} / \mathrm{v})$, $1 \%$ glucose $(\mathrm{w} / \mathrm{v}), 20 \%$ sucrose $(\mathrm{w} / \mathrm{v})$ and $0.8 \%$ agar pow$\operatorname{der}(\mathrm{w} / \mathrm{v})$ ] supplemented with $200 \mathrm{ppm}$ hygromycin B.

Phylogenetic analysis of MoHDA1 and its homologs. All nucleotide and amino acid sequences were retrieved from National Center for Biotechnology Information (NCBI) (Supplementary Table 1). The amino acid sequences were 
aligned by using Clustal Omega at EBI server (Sievers and Higgins, 2018). For phylogenetic tree construction using the maximum likelihood, MEGA7 program was used with 500 repetitions of bootstrap. Information related to domain architecture including HDAC domain, Active site and $\mathrm{Zn}^{2+}$ ion binding site were determined by Interproscan (Mulder and Apweiler, 2008).

Phenotypic assays. Vegetative growth for individual strains was measured as a colony diameter using mycelia grown in complete agar medium and oatmeal agar medium for 9 days.

Spores were harvested from 9-day-old oatmeal agar medium using a sterilized platinum loop, and suspended in $5 \mathrm{ml}$ of sterilized distilled water. The number of spores was enumerated using a hemacytometer under a light microscope. For appressorium formation assay, $40 \mu \mathrm{l}(2$ $\times 10^{5}$ spores $/ \mathrm{ml}$ ) of spore suspension was dropped on an artificial hydrophobic surface (cover slip) and then incubated in a $25^{\circ} \mathrm{C}$ moistened box for $8 \mathrm{~h}$ and $12 \mathrm{~h}$. Following incubation on plastic coverslips, the germination rate and appressorium formation rate were observed under a light microscope. The germination rate was calculated by dividing the number of germinated spores by the number of total spores. The rate of appressorium formation was calculated by dividing the number of spores forming appressorium by the number of germinated spores.

Rice plants (Oryza sativa cv. Nakdong) were used in three- to four-leaf stages grown at $25^{\circ} \mathrm{C}$ for 3 weeks for all pathogenicity assays. To carry out wound inoculation, 20 $\mu \mathrm{l}$ of spore suspension $\left(5 \times 10^{4}\right.$ spores $\left./ \mathrm{ml}\right)$ was dropped on the damaged rice leaves with a pipette tip and incubated at $25^{\circ} \mathrm{C}$ in a moisture box. For spray inoculation, $10 \mathrm{ml}$ of spore suspension $\left(5 \times 10^{4}\right.$ spores $\left./ \mathrm{ml}\right)$ was sprayed onto leaves of rice seedlings. The inoculated seedlings were placed in a moisture box for $24 \mathrm{~h}$ under dark conditions, and transferred to incubator at $25^{\circ} \mathrm{C}$ with a $16 \mathrm{~h}$ photoperiod with light. All lesions were evaluated by pixels of its area on the 5 days post inoculation.

Targeted gene deletion and complementation. Fusion PCR was used to generate knockout construct for MoHDAl gene (Yu et al., 2004). The flanking sequences of MoHOS2 (longer than $1 \mathrm{~kb}$ ) were PCR-amplified and then fused with a hygromycin B phosphotransferase $(H P H)$ cassette using a double-joint PCR. The fused construct was amplified with nested primers (Supplementary Table 2). The resulting knockout construct was then introduced into the KJ201 protoplast. Hygromycin-resistant transformants were screened first in TB3 agar medium supplemented with hygromycin B (200 ppm final concentration) and then PCR-screened for $5^{\prime}$ and $3^{\prime}$ flanking regions. Inverse PCR was used to confirm correct replacement of the gene with our construct (Park et al., 2014). After digesting genomic DNA with KpnI, the digest was allowed to self-ligate. The ligation samples were PCR-amplified using primers (Supplementary Table 2) as shown in Supplementary Fig. 1A. For complementation, the entire MoHDAl gene including the promotor region was amplified using genomic DNA of KJ201 as template. This fragment was co-transformed into protoplast from $\Delta M o h d a 1$ along with the geneticin resistant gene (neomycin phosphotransferase) fragment amplified from pII99. The complemented strains were screened first for geneticin resistance (400 ppm final concentration) and confirmed again by PCR and phenotype recovery.

Measurement of histone acetylation level at $\mathrm{H} 3 \mathrm{~K} 9$ and H3K14. Global acetylation of histone H3K9 and H3K14 was measured colorimetrically using EpiQuik Global Acetyl Histone H3-K9/K14 Quantification kits (Epigentek Group Inc., Farmingdale, NY, USA) according to the manufacturer's instruction. Luminescence was measured with a SPARK 10 M multimode micro-plate reader (Tecan Group Ltd., Männedorf, Switzerland).

Nucleic acid manipulation and expression analysis. The genomic DNA used for inverse PCR or restriction enzyme digestion was prepared by the standard method (Sambrook and Russell, 2001). The DNA used for screening with PCR was extracted using the quick method as described previously (Chi et al., 2009).

RNA was isolated from frozen fungal hyphae using MiniBEST Universal RNA Extraction kit (TaKaRa, Shiga, Japan). Quantitative real-time PCR (qRT-PCR) was performed on StepOnePlus Real-Time PCR system machine (Applied Biosystems, Foster City, CA, USA) for 40 cycles using a mixture containing $10 \mu \mathrm{l}$ of Power SYBR Green PCR Master Mix (Applied Biosystems), $2 \mu \mathrm{l}(50 \mathrm{ng} / \mu \mathrm{l})$ of cDNA template and $2 \mu \mathrm{l}$ of forward/reverse primer (Supplementary Table 3). Beta-tubulin gene was used as internal reaction control to normalize mRNA levels between different samples. The cDNA used was synthesized using $1 \mu \mathrm{g}$ of RNA extracted with the method described above, oligo dT primer, and GoTaq Real-Time PCR Systems Kit (Promega, Madison, WI, USA).

Overexpression of $\mathrm{MoCON6}$. For overexpression of MoCON6, the trpC promotor of Aspergillus nidulans and MoCON6 gene were amplified using the specific primer sets (Supplementary Table 4). Then, the amplified frag- 
ments were fused using the restriction recognition site of HindIII. This construct was co-transformed into protoplast from KJ201 with the $H P H$ cassette fragment amplified from $\mathrm{pCB} 1004$. The transformants were screened first for hygromycin B resistance (200 ppm) and confirmed again by PCR.

\section{Results and Discussion}

Phylogenetic and sequence analysis of MoHDA1. A gene (MGG_01076) encoding an ortholog of S. cerevisiae Hda1 in the genome of M. oryzae was identified by a web- based database, dbHiMo (http://hme.riceblast.snu.ac.kr) (Choi et al., 2015), and designated as MoHDA1. Pairwise local alignment using EMBOSS server (https://www.ebi. ac.uk/Tools/emboss/) revealed that MoHDA1 exhibits high similarity to Hda1 from $S$. cerevisae (52.8\%), HosA from A. nidulans (57.6\%) and Clr3 from S. pombe (51.6\%). Phylogenetic analysis of class I, II, and III HDACs including MoHDA1 clearly showed that MoHDA1 belongs class II HDAC together with MoHOS3 (Fig. 1A). In mammals, the orthologues of yeast Hdal have long N-terminal region harboring myocyte enhancer factor-2 (MEF-2)-binding motifs and 14-3-3 binding motifs. This is consistent with
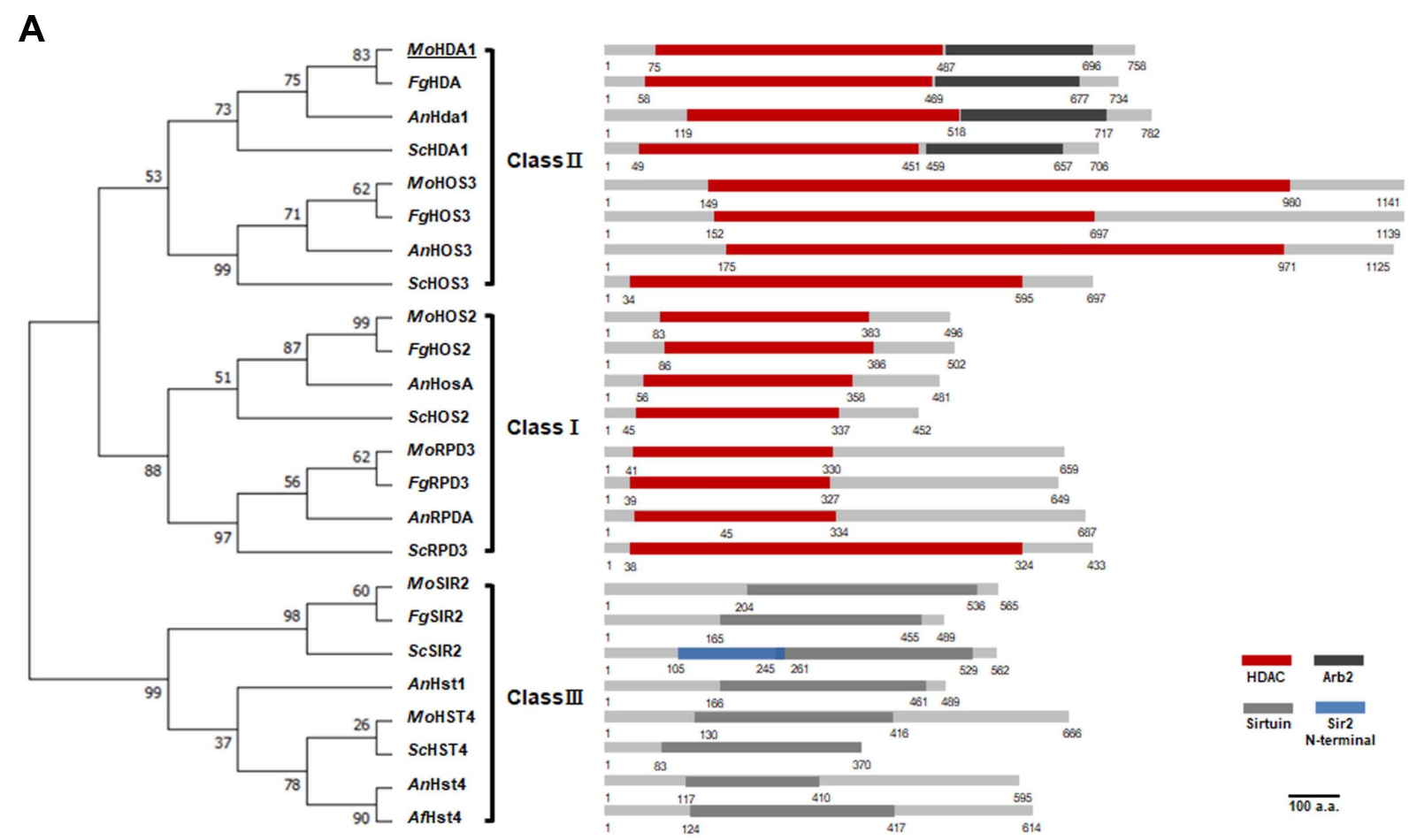

B

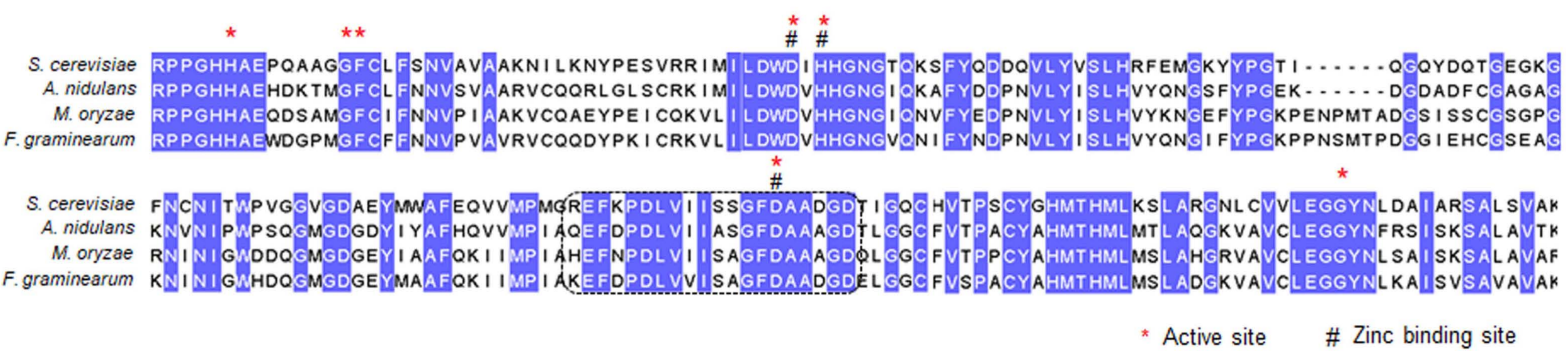

Fig. 1. Analysis of phylogenetic relationships, domain architecture, and motifs among histone deacetylases (HDACs). (A) MoHDA1 (underlined) was compared phylogenetically to class I, II, and III HDACs. Phylogenetic tree was constructed using neighbor-joining method in MEGA7 program. The numbers indicate bootstrap values. Domain architecture of proteins were shown in parallel to the tree. (B) Multiple amino acid sequence alignment among a selected set of MoHDA1 orthologs. Conserved active sites, $\mathrm{Zn}^{2+}$ binding sites and ER motif are marked by red asterisks, black hashes, and dotted box, respectively. Information on proteins used in phylogenetic tree construction is provided in Supplementary Table 1. 
the fact that they regulate cytoplasmic processes or function as signal transducers that shuttle between the cytoplasm and nucleus (Yang and Seto, 2008). In contrast to the mammalian class II HDACs, fungal counterparts do not have long N-terminal sequences. Instead, Hda1 orthologs at their C-termini possess Arb2 domain, which is predicted to act as an anchor connecting the HDAC activity of $\mathrm{Clr} 3$ to the SHREC (Snf2/Hdac Repressive) complex in fission yeast for gene silencing in heterochromatin (Job et al., 2016). The presence of well-conserved Arb2 domain in MoHDA1 points to the possibility that MoHDA1 might participate in heterochromatin formation as well.

Multiple sequence alignment confirmed that active sites and $\mathrm{Zn}^{2+}$ binding sites, all of which are required for HDAC activity (Adachi et al., 2002), are conserved in MoHDA1 (Fig. 1B). We also found that ER motifs, key residues involved in HDAC activity, were aligned almost perfectly between MoHDA1 and S. cerevisiae Hda1, suggesting that MoHDA1 is highly likely to have HDAC activity (Fig. 1B, dotted box).

Deletion of MoHDA1 and analysis of HDAC activity. In order to elucidate roles of MoHDA1 genes in fungal development and pathogenesis, we first generated deletion mutant strain for MoHDA1 gene via transformation of protoplast with knockout construct using $H P H$ cassette
A

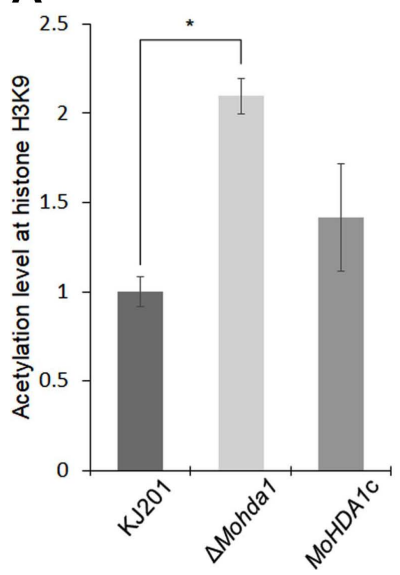

B

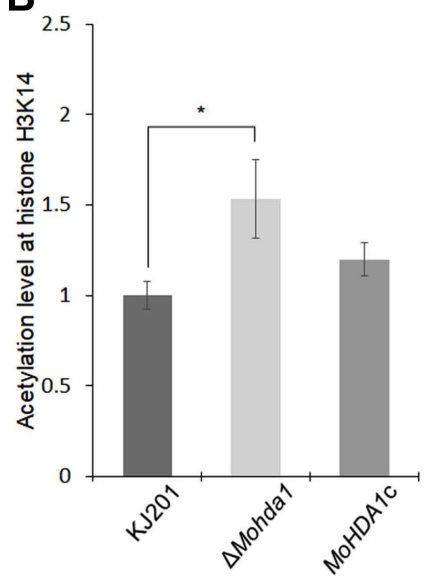

Fig. 2. Impact of $M o H D A 1$ gene deletion on histone deacetylase (HDAC) activity. (A) Histone acetylation levels at H3K9 were measured for $\triangle M O H D A 1$ and MoHDAlc, relative to the level of the wild-type. (B) Histone acetylation levels at H3K14 were measured for $\triangle M o H D A 1$ and $M o H D A 1 c$, relative to the level of the wild-type. Experiments were repeated independently three times. Error bars indicate standard deviation. Asterisk indicates significant differences in mean values, compared to the wild-type $\left({ }^{*} P<\right.$ 0.05 in Tukey's honestly significant difference test). as a selection marker (Supplementary Fig. 1A). Correct replacement of the gene with $H P H$ cassette was confirmed for only a single mutant by PCR-based screen and inverse PCR method (Park et al., 2014) (Supplementary Fig. 1B). Complementation strain was prepared by introducing a wild-type copy of MoHDAl gene under native promoter back into the deletion mutant.

In $S$. cerevisiae, it is known that Hdal can remove acetyl groups from $\mathrm{H} 3 \mathrm{~K} 9$ and $\mathrm{H} 3 \mathrm{~K} 14$ among target sites $(\mathrm{Wu}$ et al., 2001). To check whether MoHDAl gene encodes a HDAC, we measured histone acetylation levels for H3K9 and $\mathrm{H} 3 \mathrm{~K} 14$ in cell lysates extracted from the wild-type strain, deletion mutant, and complementation strain. Our HDAC activity assay showed nearly 2 -fold and 1.5 -fold increase in total cellular H3K9ac and H3K14ac levels for $\triangle M o h d a 1$, compared to the wild-type, while complementation strain showed $\sim 40$ and $20 \%$ increases in H3K9ac and H3K14ac, respectively, which did not differ significantly from acetylation level of wild-type strain (Fig. 2). Larger

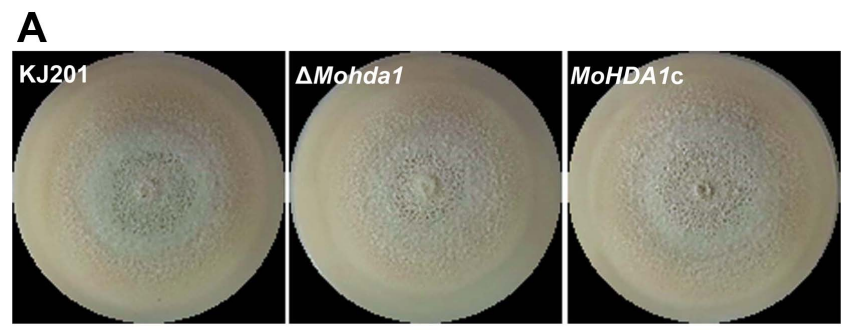

B

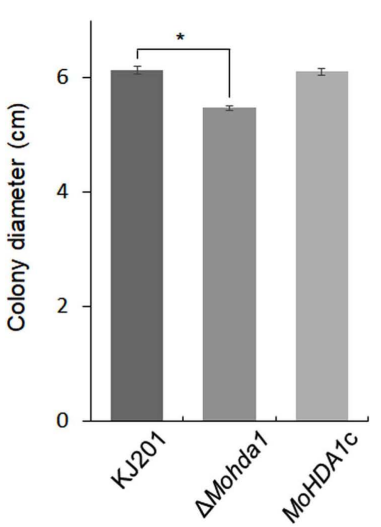

C

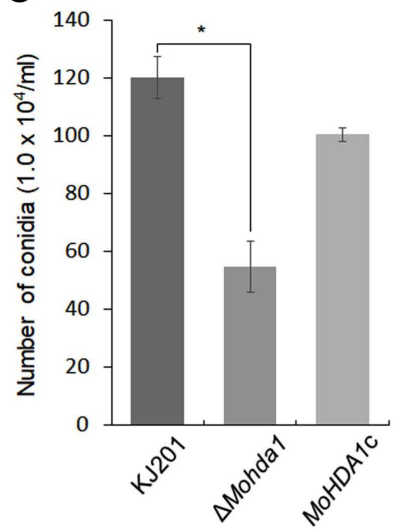

Fig. 3. Vegetative growth and asexual development of the $\Delta M o h d a 1$ strain. (A) Vegetative growth of the wild-type, $\triangle M o h d a 1$ and complementation strain (MoHDAlc) was compared on oatmeal agar plates. (B) Diameter of colonies were measured at 9 days post inoculation. (C) Number of conidia produced by each strain was counted. Error bars indicate standard deviation. Asterisk indicates significant differences in mean values, compared to the wild-type ( ${ }^{*} P<0.05$ in Tukey's honestly significant difference test). 
increase of $\mathrm{H} 3 \mathrm{~K} 9$ acetylation level in the mutant may suggest that MoHDA1 has more preference on H3K9 than $\mathrm{H} 3 \mathrm{~K} 14$ as target sites, and that genes having increased acetylation levels in the mutant are likely to be mis-regulated. It is therefore logical to infer from this result that MoHDA1 is a bona-fide HDAC in M. oryzae, since lack of MoHDA1 genes leads to increases in acetylation levels at H3K9 and H3K14. Together with the presence of Arb2 domain at its $\mathrm{C}$-terminus, our results suggest that heterochromatin formation and regulation of gene transcription would be disrupted in the mutant lacking MoHDA1.

Effect of MoHDA1 deletion on fungal growth and asexual development. Compared to the wild-type strain KJ201, the deletion mutant showed a little reduction in radial growth $(\sim 10 \%)$ (Fig. 3A and B). However, the mutant showed no difference in colony morphology and pigmentation, compared to wild-type and complementation strain (Fig. 3A). When asexual spore production from the mutant was examined, we found that the mutant is severely defective in asexual sporulation (Fig. 3C), although morphology of mutant conidia was comparable to that of wild-type (Fig. 5A). These data suggest that MoHDA1-mediated histone deacetylation is mainly required for asexual reproduction in M. oryzae.

Transcription of genes involved in asexual reproduction. Based on large reduction in asexual spore production of the mutant, we hypothesized that MoHDA1 regulates transcription of genes implicated in conidiogenesis. To investigate this possibility, we examined relative transcript abundance of a set of genes that are known to be involved in asexual reproduction in $M$. oryzae, using qRT-PCR. Instead of using RNAs from submerged cultures, we extracted RNAs from plate culture conditions that promote asexual development (oatmeal agar plate culture under constant fluorescent light and aeration at $25^{\circ} \mathrm{C}$ ), in an attempt to capture the aberrant changes in transcription of conidiation-related genes during sporulation in the mutant. Under this condition, we compared the transcript abundances of a set of the conidiation-related genes including MoACR1, MoFLBC, MoFLBD, MoCON8, and MoHOX2 (Supplementary Fig. 2). On the contrary to our expectation, qRT-PCR analysis showed that all the genes except MoCON6 showed no difference in relative transcript abundance among strains. The MoCON6 gene expression exhibited 3-fold up-regulation in the mutant, compared to the wild-type (Supplementary Fig. 2).

Since MoHDAl encodes a HDAC, its deletion is, in general, expected to increase histone acetylation level and as a result, transcription of its target genes. Therefore, it is possible that MoCON6 would be one of the targets that are regulated by MoHDA1-mediated histone deacetylation, although we cannot rule out the possibility that MoCON6 is up-regulated via indirect mechanism(s). Based on our observation regarding MoCON6 induction in the mutant, we asked if we could establish the mechanical link between up-regulation of MoCON6 gene and reduction in asexual sporulation.

To test this, we made a construct that over-expresses $\mathrm{Mo}$ CON6, and introduced it into the wild-type strain. Following PCR-based screening for the presence of the construct in the genome, a few transformants carrying the construct was selected and further examined by qRT-PCR for overexpression of MoCON6. Interestingly, transcription of MoCON6 gene showed astronomical increase in the transformants, for which over-expression were confirmed by qRT-PCR (\#85 and 86 in Fig. 4A). This result was highly reproducible throughout several rounds of independent experiments, strongly suggesting that it is not experimental artefacts. When transcription level of MoCON6 and asexual spore production were compared across different strains, we were able to see the negative correlation between the two results (Fig. 4A and B). In a previous work, it was shown that a mutation in MoCON6 could completely

A
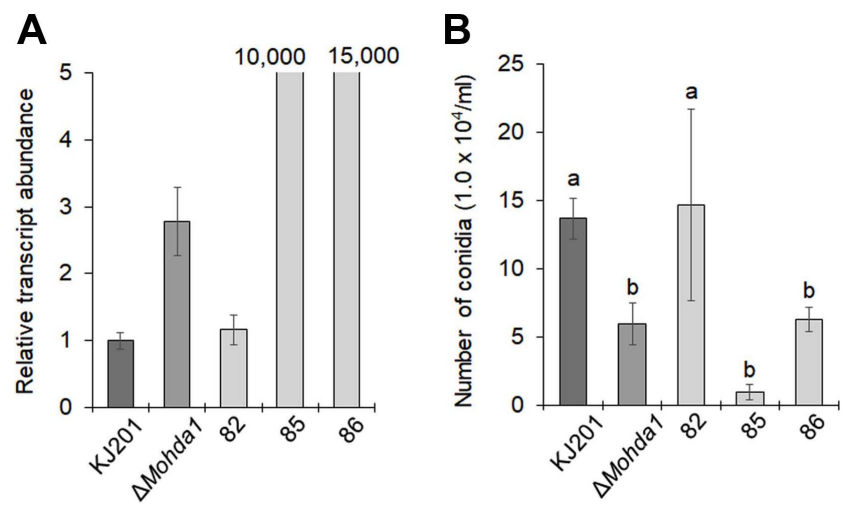

Fig. 4. Analysis of MoCON6 expression level and its effect on conidiation in the wild-type, $\triangle M o h d a 1$ and over-expression lines ( 85 and $86 ; 82$ is a strain having over-expression construct in the genome without apparent over-expression of MoCON6). (A) Relative transcript abundance of MoCON6 gene, which is known to be important for conidiation, was measured using RNAs extracted from oatmeal plate cultures under conidiation-promoting condition. Relative transcript abundances of two over-expression strains, 85 and 86 , are indicated as numbers on top of the corresponding bars. (B) Number of conidia that are produced from each strain was enumerated. Error bars indicate standard deviation. Different alphabets indicate significant differences in mean values $(P<0.05$ in Tukey's honestly significant difference test). 
abolish conidial production in M. oryzae (Shi and Leung, 1995). In other studies, it was demonstrated that MoCON6 is up-regulated in the deletion mutant of MoMsn2 gene encoding a putative zinc-finger protein (Zhang et al., 2014). Notably, $\Delta M o m s n 2$ was severely impaired in conidial production. Based on our data and previous works, it is tempting to conjecture that there might be dosage effect on asexual reproduction by MoCON6 expression, of which proper regulation is dependent upon MoHDA1-mediated HDAC activity.

Appressorium development and pathogenicity. We next tested if $\Delta M o h d a 1$ conidia are able to undergo developmental changes that are required for pathogenesis. When the mutant spores were placed on plastic coverslip and allowed to germinate, a slight reduction in germination was observed (Fig. 5A and B). However, even in the mutant, germination rate was nearly $90 \%$, indicating that the amount of reduction is not biologically significant. Unlike

\section{A}

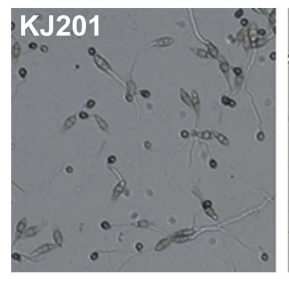

B

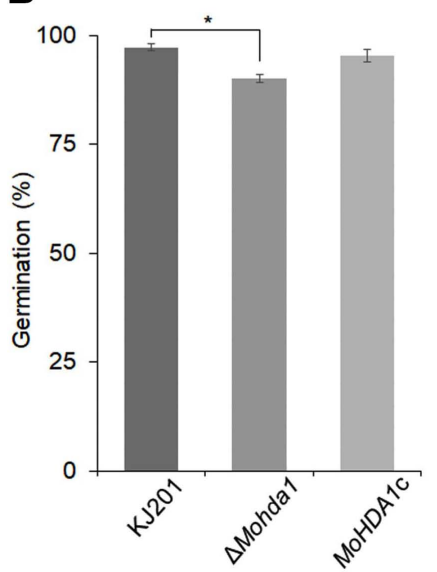

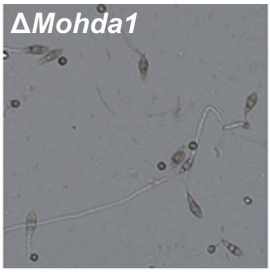

C

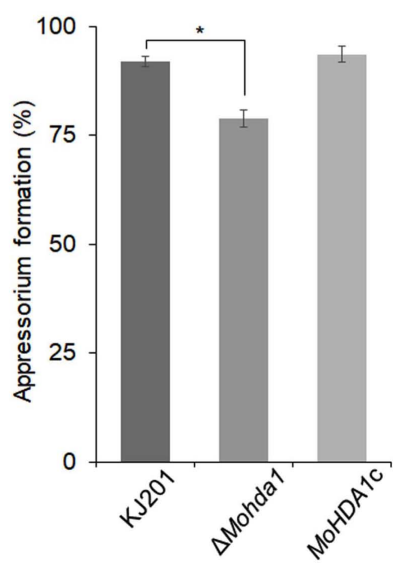

Fig. 5. Conidial germination and appressorium formation of the mutant. (A) Germination and appressorium formation were examined and compared among strains 8 hpi on plastic coverslips. (B) Germination rate was measured among the wild-type, $\triangle M o H D A 1$ and MoHDA1c. (C) Appressorium formation was measured among the wild-type, $\triangle M o H D A 1$ and MoHDA1c. Error bars indicate standard deviation. Asterisk indicates significant differences in mean values, compared to the wild-type $\left({ }^{*} P<0.05\right.$ in Tukey's honestly significant difference test). germination, considerable decrease in appressorium formation was observed for the mutant (Fig. 5A and C). Compared to the wild-type showing higher than $90 \%$ appressorium formation rate, only $\sim 75 \%$ of germinated conidia of mutant developed appressoria (Fig. 5C), suggesting that $M o H D A 1$ is required for efficient appressorium formation.
A

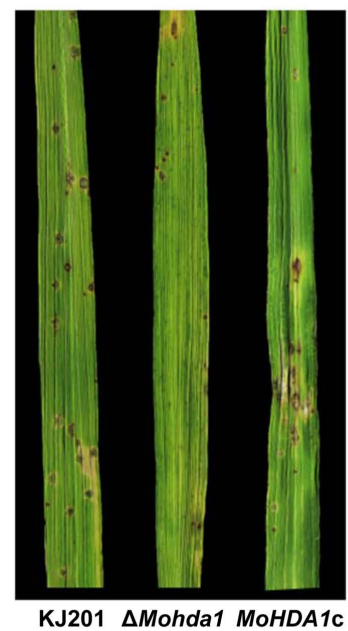

C

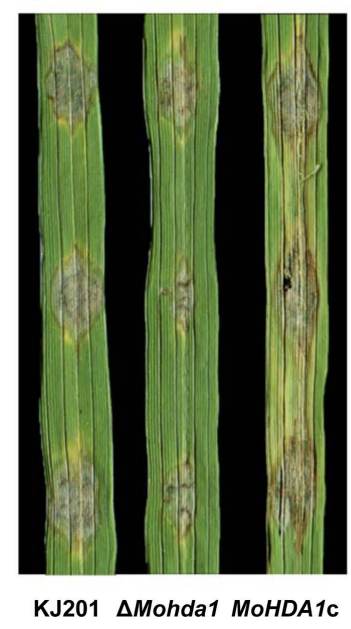

B

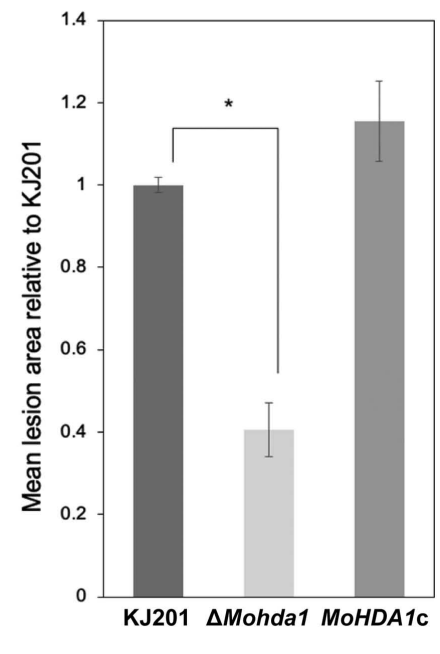

D

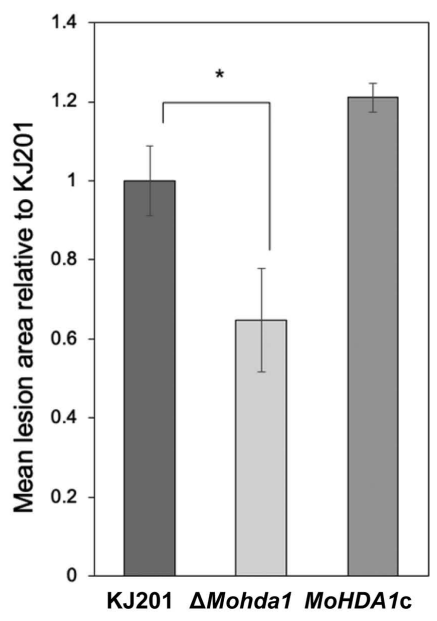

Fig. 6. Pathogenicity of $\triangle M o h d a l$ was compared to that of wildtype and complementation strain. (A) Rice leaves were sprayinoculated with conidial suspensions and evaluated for development of disease symptoms 7 dpi. (B) Lesion areas were measured by image analysis and used for comparison of disease severity. (C) Ability to grow inside plant (invasive growth) was evaluated by wound-inoculation, through which spore suspensions $(2 \times$ $10^{5}$ spores $/ \mathrm{ml}$ ) are placed upon wound-sites to allow entry of the fungus into plants without appressorium. (D) Lesion areas were measured by image analysis and used for comparison of disease severity. Error bars indicate standard deviation. Asterisk indicates significant differences in mean values, compared to the wild-type $(*<0.05$ in Tukey's honestly significant difference test). 
When conidia of $\Delta M o h d a 1$ were sprayed onto the susceptible rice plants, the mutant was able to cause disease symptoms, but produced lesions less in number and smaller in size than the wild-type and complementation strain, indicating that $M o H D A 1$ is required for full virulence of the fungus (Fig. 6A and B). In an attempt to explain such decrease in virulence, we examined invasive growth of the mutant by wound inoculation method in which spore suspension is placed upon the wound site made by pipet tip. This allows the fungus to gain access to the plant cells without appressorium-mediated penetration. The woundinoculation experiment showed that the wild-type and complementation strain can cause typical blast lesion development at 5 days post inoculation (dpi), whereas the mutant developed smaller lesions that measure approximately $60 \%$ of wild-type lesions, indicating that the mutant is compromised in its ability to grow inside the plants (Fig. 6C and D).

In order to dissect the defect in pathogenicity of $\triangle M O$ hdal, we first checked out the ability of the mutant to penetrate into plant cell using onion epidermal cell layers. This experiment showed that the mutant is rarely able to penetrate plant cells, and it developed poorly growing invasive hyphae when it succeeded in penetration (Supplementary Fig. 3). Next, we tested if the mutant is capable of coping with ROS or cell-wall degrading enzymes by supplementing the media with hydrogen peroxide and lysing enzyme separately or in combination (Supplementary Fig. 4). This experiment showed that the mutant is comparable to the wild-type in its sensitivity to the treatment with $\mathrm{H}_{2} \mathrm{O}_{2}$ and lysing enzyme (Supplementary Fig. 4). These results suggest that the observed reduction in virulence of the mutant is attributable to the defects in appressorium formation, penetration into host plants, and invasive growth. Considering the roles of MoHDA1 as a HDAC in gene silencing and heterochromatin formation, whether proper transcription of genes encoding effectors in M. oryzae is dependent on MoHDA1 function warrant future study.

\section{Acknowledgments}

This work was supported by 2018 Yeungnam University Research Grant (218A380038) and a grant from the Nakdonggang National Institute of Biological Resources (NNIBR), funded by the Ministry of Environment (MOE) of the Republic of Korea (NNIBR202002101).

\section{Electronic Supplementary Material}

Supplementary materials are available at The Plant Pathology Journal website (http://www.ppjonline.org/).

\section{References}

Adachi, N., Kimura, A. and Horikoshi, M. 2002. A conserved motif common to the histone acetyltransferase Esal and the histone deacetylase Rpd3. J. Biol. Chem. 277:35688-35695.

Bannister, A. J. and Kouzarides, T. 2011. Regulation of chromatin by histone modifications. Cell Res. 21:381-395.

Carmen, A. A., Rundlett, S. E. and Grunstein, M. 1996. HDA1 and HDA3 are components of a yeast histone deacetylase (HDA) complex. J. Biol. Chem. 271:15837-15844.

Chi, M.-H., Park, S.-Y. and Lee, Y.-H. 2009. A quick and safe method for fungal DNA extraction. Plant Pathol. J. 25:108111.

Choi, J., Kim, K.-T., Huh, A., Kwon, S., Hong, C., Asiegbu, F. O., Jeon, J. and Lee, Y.-H. 2015. dbHiMo: a web-based epigenomics platform for histone-modifying enzymes. Database (Oxford) 2015:bav052.

Dean, R., Van Kan, J. A. L., Pretorius, Z. A., Hammond-Kosack, K. E., Di Pietro, A., Spanu, P. D., Rudd, J. J., Dickman, M., Kahmann, R., Ellis, J. and Foster, G. D. 2012. The Top 10 fungal pathogens in molecular plant pathology. Mol. Plant Pathol. 13:414-430.

Ding, S.-L., Liu, W., Iliuk, A., Ribot, C., Vallet, J., Tao, A., Wang, Y., Lebrun, M.-H. and Xu, J.-R. 2010. The tig1 histone deacetylase complex regulates infectious growth in the rice blast fungus Magnaporthe oryzae. Plant Cell 22:2495-2508.

Elias-Villalobos, A., Barrales, R. R. and Ibeas, J. I. 2019. Chromatin modification factors in plant pathogenic fungi: insights from Ustilago maydis. Fungal Genet. Biol. 129:52-64.

Elias-Villalobos, A., Fernández-Álvarez, A., Moreno-Sánchez, I., Helmlinger, D. and Ibeas, J. I. 2015. The Hos2 histone deacetylase controls Ustilago maydis virulence through direct regulation of mating-type genes. PLoS Pathog. 11:e1005134.

Fernandez, J. and Orth, K. 2018. Rise of a cereal killer: the biology of Magnaporthe oryzae biotrophic growth. Trends Microbiol. 26:582-597.

Howard, R. J. and Valent, B. 1996. Breaking and entering: host penetration by the fungal rice blast pathogen Magnaporthe grisea. Annu. Rev. Microbiol. 50:491-512.

Jeon, J., Kwon, S. and Lee, Y.-H. 2014. Histone acetylation in fungal pathogens of plants. Plant Pathol. J. 30:1-9.

Jiang, C., Zhang, X., Liu, H. and Xu, J.-R. 2018. Mitogenactivated protein kinase signaling in plant pathogenic fungi. PLoS Pathog. 14:e1006875.

Job, G., Brugger, C., Xu, T., Lowe, B. R., Pfister, Y., Qu, C., Shanker, S., Sanz, J. I. B., Partridge, J. F. and Schalch, T. 2016. SHREC silences heterochromatin via distinct remodeling and deacetylation modules. Mol. Cell 62:207-221.

Lee, J., Lee, J.-J. and Jeon, J. 2019. A histone deacetylase, MoHOS2 regulates asexual development and virulence in the rice blast fungus. J. Microbiol. 57:1115-1125.

Lee, K. K. and Workman, J. L. 2007. Histone acetyltransferase complexes: one size doesn't fit all. Nat. Rev. Mol. Cell Biol. 
8:284-295.

Li, Y., Wang, C., Liu, W., Wang, G., Kang, Z., Kistler, H. C. and $\mathrm{Xu}, \mathrm{J} .-\mathrm{R} .2011$. The HDF1 histone deacetylase gene is important for conidiation, sexual reproduction, and pathogenesis in Fusarium graminearum. Mol. Plant-Microbe Interact. 24:487-496.

Mulder, N. J. and Apweiler, R. 2008. The InterPro database and tools for protein domain analysis. Curr. Protoc. Bioinformatics Chapter 2:Unit 2.7.

Park, J., Kim, S., Kwon, S. and Lee, Y.-H. 2014. A quick and accurate screening method for fungal gene-deletion mutants by direct, priority-based, and inverse PCRs. J. Microbiol. Methods 105:39-41.

Robbins, N., Leach, M. D. and Cowen, L. E. 2012. Lysine deacetylases Hda1 and Rpd3 regulate Hsp90 function thereby governing fungal drug resistance. Cell Rep. 2:878-888.

Rundlett, S. E., Carmen, A. A., Kobayashi, R., Bavykin, S., Turner, B. M. and Grunstein, M. 1996. HDA1 and RPD3 are members of distinct yeast histone deacetylase complexes that regulate silencing and transcription. Proc. Natl. Acad. Sci. U. S. A. 93:14503-14508.

Ryder, L. S. and Talbot, N. J. 2015. Regulation of appressorium development in pathogenic fungi. Curr. Opin. Plant Biol. 26:8-13.

Sambrook, J. and Russell, D. W. 2001. Molecular cloning: a laboratory manual. 3rd ed. Vol. 3. Cold Spring Harbor Laboratory Press, Cold Spring Harbor, NY, USA. 2100 pp.

Seto, E. and Yoshida, M. 2014. Erasers of histone acetylation: the histone deacetylase enzymes. Cold Spring Harb. Perspect. Biol. 6:a018713.

Shen, H., Zhu, Y., Wang, C., Yan, H., Teng, M. and Li, X. 2016. Structural and histone binding ability characterization of the ARB2 domain of a histone deacetylase Hda1 from Saccharomyces cerevisiae. Sci. Rep. 6:33905.

Shi, Z. and Leung, H. 1995. Genetic analysis of sporulation in
Magnaporthe grisea by chemical and insertional mutagenesis. Mol. Plant-Microbe Interact. 8:949-959.

Sievers, F. and Higgins, D. G. 2018. Clustal Omega for making accurate alignments of many protein sequences. Protein Sci. 27:135-145.

Srikantha, T., Tsai, L., Daniels, K., Klar, A. J. and Soll, D. R. 2001. The histone deacetylase genes HDA1 and RPD3 play distinct roles in regulation of high-frequency phenotypic switching in Candida albicans. J. Bacteriol. 183:4614-4625.

Talbot, N. J. 2003. On the trail of a cereal killer: exploring the biology of Magnaporthe grisea. Annu. Rev. Microbiol. 57:177202.

Wilson, R. A. and Talbot, N. J. 2009. Under pressure: investigating the biology of plant infection by Magnaporthe oryzae. Nat. Rev. Microbiol. 7:185-195.

Wu, J., Suka, N., Carlson, M. and Grunstein, M. 2001. TUP1 utilizes histone $\mathrm{H} 3 / \mathrm{H} 2 \mathrm{~B}$-specific HDA1 deacetylase to repress gene activity in yeast. Mol. Cell 7:117-126.

Yang, X.-J. and Seto, E. 2008. The Rpd3/Hda1 family of lysine deacetylases: from bacteria and yeast to mice and men. Nat. Rev. Mol. Cell Biol. 9:206-218.

Yu, J.-H., Hamari, Z., Han, K.-H., Seo, J.-A., Reyes-Domínguez, Y. and Scazzocchio, C. 2004. Double-joint PCR: a PCRbased molecular tool for gene manipulations in filamentous fungi. Fungal Genet. Biol. 41:973-981.

Zacchi, L. F., Schulz, W. L. and Davis, D. A. 2010. HOS2 and HDA1 encode histone deacetylases with opposing roles in Candida albicans morphogenesis. PLoS ONE 5:e12171.

Zhang, H., Zhao, Q., Guo, X., Guo, M., Qi, Z., Tang, W., Dong, Y., Ye, W., Zheng, X., Wang, P. and Zhang, Z. 2014. Pleiotropic function of the putative zinc-finger protein MoMsn2 in Magnaporthe oryzae. Mol. Plant-Microbe Interact. 27:446-460.

Zhang, S. and Xu, J.-R. 2014. Effectors and effector delivery in Magnaporthe oryzae. PLoS Pathog. 10:e1003826. 